\title{
Real-Time Monitoring of Fibrinogen Cross-Linking on Model Biomaterial Surfaces with Quartz Crystal Microbalance
}

\author{
Brian C. Heinze and Jeong-Yeol Yoon*
}

Department of Agricultural and Biosystems Engineering, The University of Arizona, Tucson, Arizona 85721-0038, USA

\begin{abstract}
A quartz crystal microbalance was used for real-time monitoring of fibrinogen cross-linking on three model biomaterial surfaces. Fibrinogen adsorbs slowly and forms a less rigid multi-layer on hydrophobic surfaces, while it adsorbs quickly, forming a single mono-layer on hydrophilic surfaces. The extent of fibrinogen cross-linking is greater on hydrophobic surfaces. Fibrinogen cross-linking can also rigidify the relatively soft coatings of poly(methyl methacrylate) and dodecanethiol self-assembled monolayer.
\end{abstract}

\section{INTRODUCTION}

Whenever a biomaterial comes into contact with blood, wound healing begins to occur. The first stage of this wound healing is blood coagulation, as the protein fibrinogen is adsorbed and cross-linked into a fibrin polymer, thus forming a clot [1]. Extensive blood coagulation on biomaterials is generally undesirable, such that it is expedient when designing a device to choose a material that will minimize the rate of clot formation. The current method of choice for monitoring immobilized proteins in their binding activities is surface plasmon resonance (SPR) spectroscopy [2]; however, the initial equipment and replacement chips are relatively expensive, and the surfaces of the chips are somewhat difficult to modify [3]. It is also inappropriate for monitoring blood coagulation, which is primarily an issue of structural change, rather than an issue of adsorption/adhesion [1]. The quartz crystal microbalance (QCM) is considered to be a better alternative over SPR for monitoring blood coagulation, as the QCM is relatively inexpensive, and the surfaces of the crystals are easily modified, for example, through spin coating. This method makes it possible to monitor the amount of fibrinogen adsorbed onto a given surface, and subsequently monitor the rate of fibrin polymerization (clot formation) through analysis of the changes in frequency and resistance of the piezoelectric quartz crystal over time, while in contact with the final proteins of the blood coagulation cascade.

The frequency change of the QCM is related to the adsorbed mass. In fact, the exact mass can be calculated from the frequency change $(\Delta f)$ with Sauerbrey equation if the adsorbed mass is ideally rigid [4]. The resistance change $(\Delta R)$ of the QCM is related to the rigidity (more precisely, the complex shear modulus; $G^{*}=G^{\prime}+j G^{\prime}$, where $G^{\prime}$ is the storage modulus and $G$ " is the loss modulus) as well as the mass of the adsorbed layer. Unfortunately, the equations relating $\Delta R$ to $G^{*}$ are analytically insolvable for the thin adsorbed layers [5]. Due to this complication, blood coagulation

\footnotetext{
*Address correspondence to this author at the Department of Agricultural and Biosystems Engineering, The University of Arizona, Tucson, Arizona 85721-0038, USA; E-mail: jyyoon@email.arizona.edu
}

has mostly been characterized by $\Delta f$ in the QCM analysis (fibrinogen adsorption and subsequent growth of the adsorbed layer) [6-8]. As frequency monitoring provides minimum information on the structural change of the adsorbed fibrinogen, other analyses are often accompanied, such as SPR and/or atomic force microscopy (AFM) [6-8].

Recently, QCM-D has been investigated to monitor the viscoelastic change of the adsorbed fibrinogen $[9,10]$. The change in the dissipation factor $(\Delta D)$ that is monitored can be related to the resistance change $(\Delta R)$ of the conventional QCM. Typically two different sets of experiments are performed to monitor $\Delta D$, with or without inducing crosslinking. Real-time monitoring of fibrinogen cross-linking is still a rarity in QCM analyses. In addition, the effects of surface hydrophobicity on fibrinogen cross-linking have not been investigated systematically with QCM.

\section{MATERIALS AND METHODS}

\subsection{Preparation of Model Biomaterial Surfaces}

The piezoelectric crystals used were $5 \mathrm{MHz}, 2.54 \mathrm{~cm}$ diameter, with polished gold electrodes on both sides and chromium under layers (Stanford Research Systems, Sunnyvale, CA, USA). The gold surfaces were decontaminated of dust and other possible contaminates through sonication for ten minutes followed by rinsing with deionized water. This gold surface of the QCM crystal was used without further modification as a model of a smooth hydrophilic surface.

495 poly(methyl methacrylate) (PMMA) 3\% (MicroChem Corp., Newton, MA, USA) in anisole was spun onto the pre-cleaned crystal at $500 \mathrm{rpm} / 5 \mathrm{~s}$ and 4,000 rpm / $65 \mathrm{~s}$. This crystal coated with PMMA was used to represent an intermediately rough and moderately hydrophobic surface.

Finally, a dodecanethiol self-assembled monolayer (SAM) was added to pre-cleaned gold crystals through overnight incubation in a $10 \mathrm{mM}$ solution of 1-dodecanethiol in ethanol (Sigma-Aldrich, St. Louis, MO, USA). 
The relative hydrophobicities of the above crystals were measured through contact angle analysis of a $5 \mu \mathrm{L}$ drop of water placed on top of the crystal (Fig. 1). The contact angles were measured as gold: $35 \pm 3^{\circ}$, PMMA: $62 \pm 5^{\circ}$, and dodecanethiol: $103 \pm 4^{\circ}$ (Fig. 1).

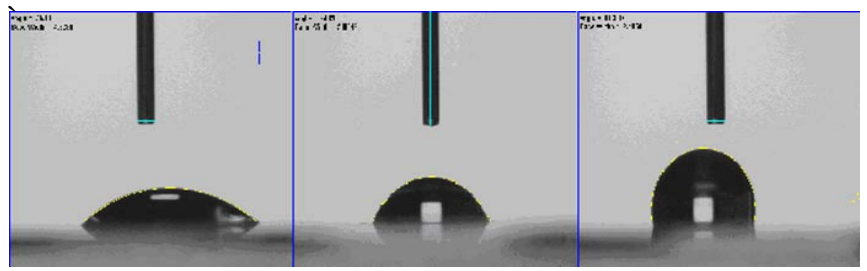

Fig. (1). Contact angle analysis of the three crystal surfaces. From left to right: gold $\left(35^{\circ}\right)$, PMMA $\left(62^{\circ}\right)$ and dodecanethiol $\left(103^{\circ}\right)$.

\subsection{Protein Solutions Preparation}

Lyophilized fibrinogen from human plasma (Calbiochem, San Diego, CA, USA) was reconstituted to a concentration of $100 \mu \mathrm{g} \cdot \mathrm{mL}^{-1}$ by dissolving in $10 \mathrm{mM} \mathrm{pH} 7.4$ phosphate buffered saline (PBS) (Sigma) at $37^{\circ} \mathrm{C}$ for approximately $30 \mathrm{~min}$, immediately prior to use. Lyophilized thrombin from human plasma (Calbiochem), coagulation factor XIII from human plasma (Calbiochem), and calcium chloride (Calbiochem) were dissolved into a second solution of PBS at concentrations of $10 \mathrm{U} \cdot \mathrm{mL}^{-1}, 10 \mu \mathrm{g} \cdot \mathrm{mL}^{-1}$, and 400 $\mu \mathrm{g} \cdot \mathrm{mL}^{-1}$, respectively.

\subsection{AFM Analysis}

Two polished gold crystals were cleaned of contamination through sonication for ten minutes followed by rinsing with deionized water. These crystals were then incubated in a $10 \mathrm{mM} \mathrm{pH}$ 7.4 PBS solution (Sigma) containing fibrinogen (Calbiochem) at a concentration of $100 \mu \mathrm{g} \cdot \mathrm{mL}^{-1}$. Subsequent to incubation, Crystals were rinsed with PBS and dried gently over nitrogen. One of these crystals was then exposed to a secondary solution of $10 \mathrm{mM} \mathrm{pH} \mathrm{7.4} \mathrm{PBS} \mathrm{(Sigma)} \mathrm{contain-}$ ing thrombin from human plasma (Calbiochem), coagulation factor XIII from human plasma (Calbiochem) and calcium chloride (Calbiochem) in concentrations of $10 \mathrm{U} \cdot \mathrm{mL}^{-1}, 10$ $\mu \mathrm{g} \cdot \mathrm{mL}^{-1}$, and $400 \mu \mathrm{g} \cdot \mathrm{mL}^{-1}$, respectively, for ten minutes before being rinsed with PBS and dried over nitrogen. Both of these crystals were then analyzed with AFM (Digital Instruments 3100, Plainview, NY, USA) in tapping mode, with silicon nitride probes (NSC-15, Mikromasch, San Jose, CA, USA).

\subsection{Analysis Procedure}

A PBS buffer solution was primarily run over the crystals at $30 \mu \mathrm{L} \cdot \mathrm{min}^{-1}$ until the crystal exhibited stable frequency and resistance measurements.

The fibrinogen solution was run over the crystal via an axial flow cell model no. O100FC (control volume $=150$ $\mu \mathrm{L}$; Stanford Research Systems) and a syringe pump model no. 780210V (KD Scientific Inc., Holliston, MA, USA) at 30 $\mu \mathrm{L} \cdot \mathrm{min}^{-1}$ until monolayer coverage of the protein was achieved. Upon monolayer coverage of fibrinogen, the thrombin/factor XIII/ $\mathrm{Ca}^{2+}$ solution was run over the crystal at the same rate. This procedure was repeated for each of the three respective surfaces: gold, PMMA, and dodecanethiol.

\section{RESULTS}

\subsection{QCM Measurements}

Upon addition of the fibrinogen solution to the flow cell, the polished gold surfaced showed an initial frequency decrease of $60 \mathrm{~Hz}$ coupled with a resistance increase of approximately $2.5 \Omega$. After addition of the thrombin/factor $\mathrm{XIII} / \mathrm{Ca}^{2+}$ solution, the crystal showed a frequency increase of $5 \mathrm{~Hz}$ coupled with a resistance decrease of $2.5 \Omega$ (Fig. 2 ).
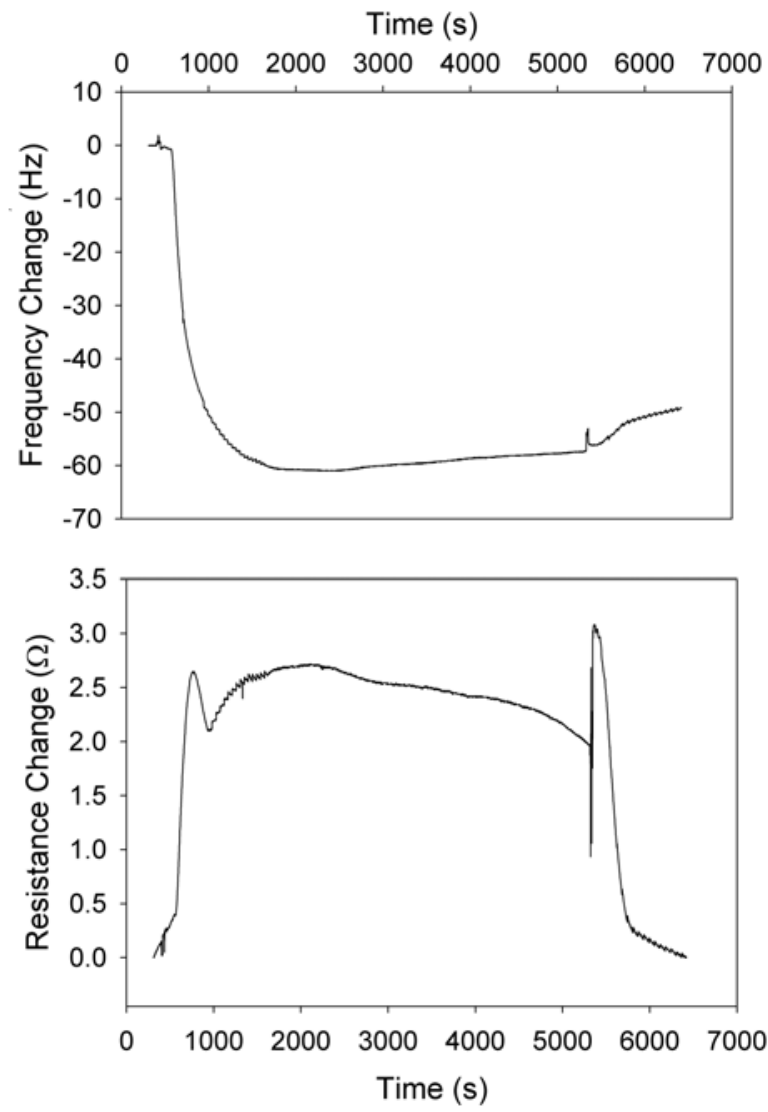

Fig. (2). Frequency change (Hz; top) and resistance change $(\Omega$; bottom) of crystal vs. time (s), with gold surface.

The Fig. (2) result is a representative of three different experiments. The mean frequency decrease was $68 \pm 7 \mathrm{~Hz}$ and the mean resistance increase was $4 \pm 1 \Omega$.

The PMMA showed an initial frequency decrease of 90 $\mathrm{Hz}$ for approximately $3,000 \mathrm{~s}$, coupled with a resistance increase of $4.0 \Omega$. Upon addition of the thrombin solution, the crystal exhibited an additional frequency decrease of $20 \mathrm{~Hz}$ coupled with a resistance decrease of $8 \Omega$ (Fig. 3).

The dodecanethiol showed an initial frequency decrease of $60 \mathrm{~Hz}$ for approximately $5,000 \mathrm{~s}$, coupled with a resistance increase of $6 \Omega$. Upon addition of the thrombin/factor $\mathrm{XIII} / \mathrm{Ca}^{2+}$ solution, the crystal exhibited an additional frequency decrease of $30 \mathrm{~Hz}$ coupled with a resistance decrease of $7 \Omega$ (Fig. 4).

Both Figs. (3 and 4$)$ are representative results; we were unable to evaluate the means and standard deviations of frequency and resistance changes as they kept decreasing against time. 

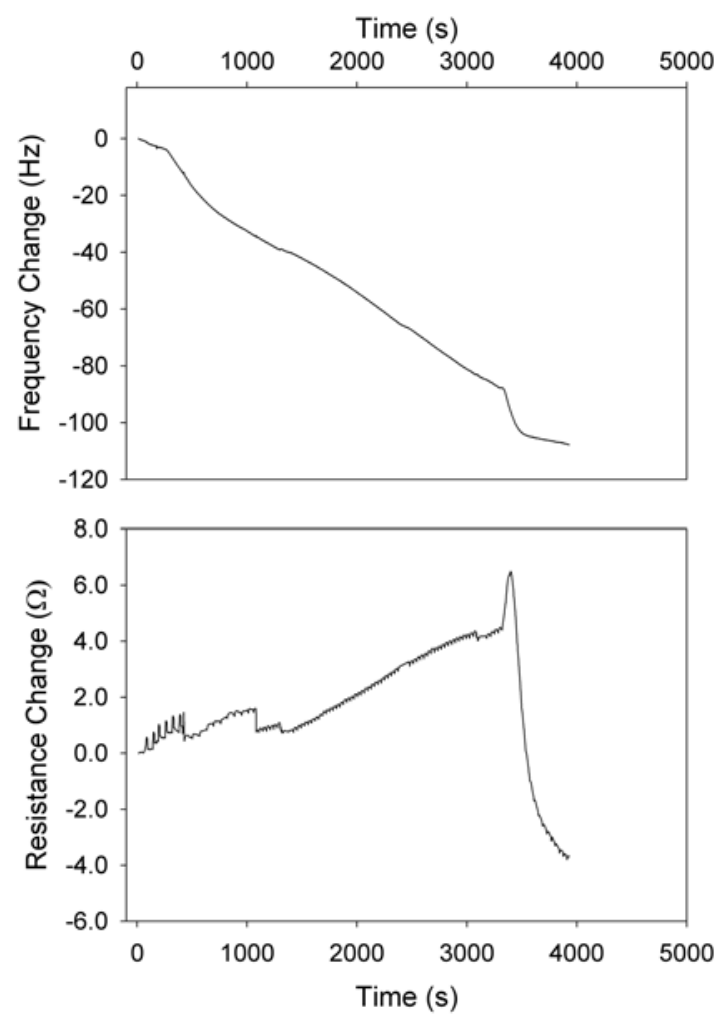

Fig. (3). (a) Top: frequency change $(\mathrm{Hz})$ and (b) bottom: resistance change $(\Omega)$ of crystal vs. time (s), with PMMA coated surface.
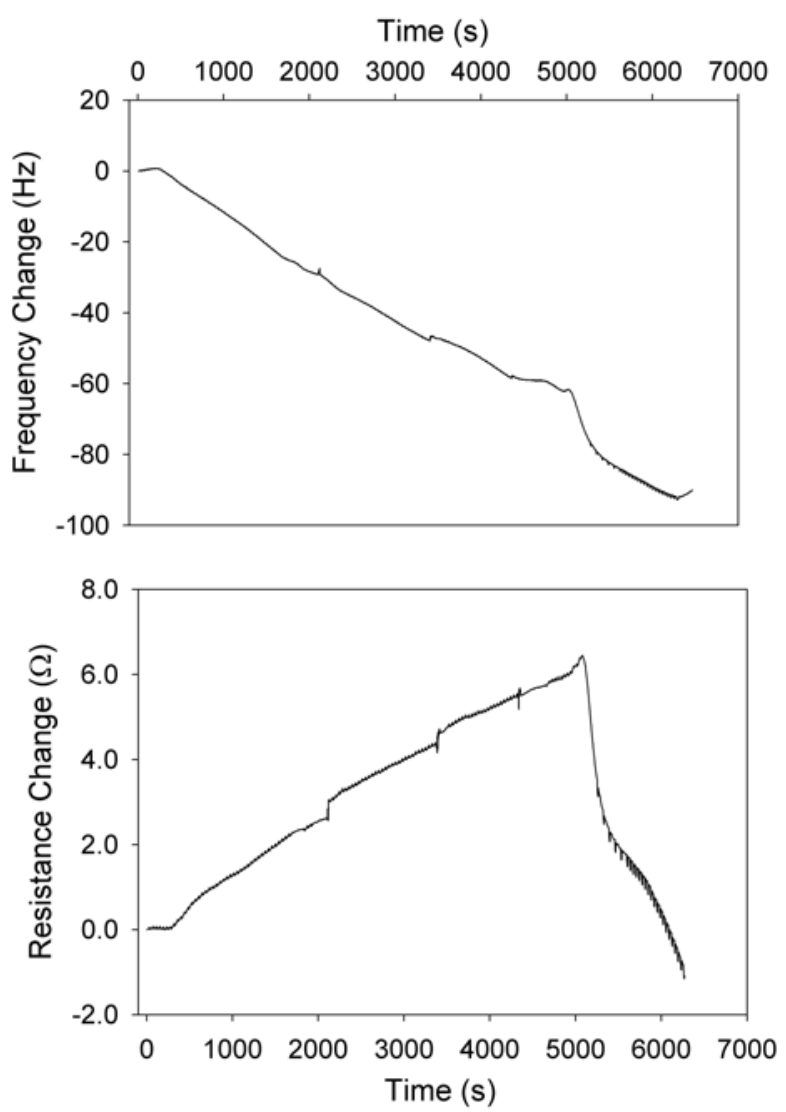

Fig. (4). (a) Top: frequency change $(\mathrm{Hz})$ and (b) bottom: resistance change $(\Omega)$ of crystal vs. time (s), with dodecanethiol.

\subsection{AFM Analysis}

Two crystal surfaces were imaged with AFM, one with adsorbed fibrinogen not exposed to thrombin, factor XIII, and calcium, and one that had been exposed. The proteins on the unexposed surface were seen to have an average size of $50-150 \mathrm{~nm}$, while the thrombin exposed protein aggregates were sized from $500 \mathrm{~nm}-2.5 \mu \mathrm{m}$ (Fig. 5). RMS roughness measurements of the three different surface modifications ( $\mathrm{Au}, \mathrm{PMMA}$, and dodecanethiol) were measured to be 3.54 $\mathrm{nm}, 0.86 \mathrm{~nm}$, and, $3.32 \mathrm{~nm}$, respectively.

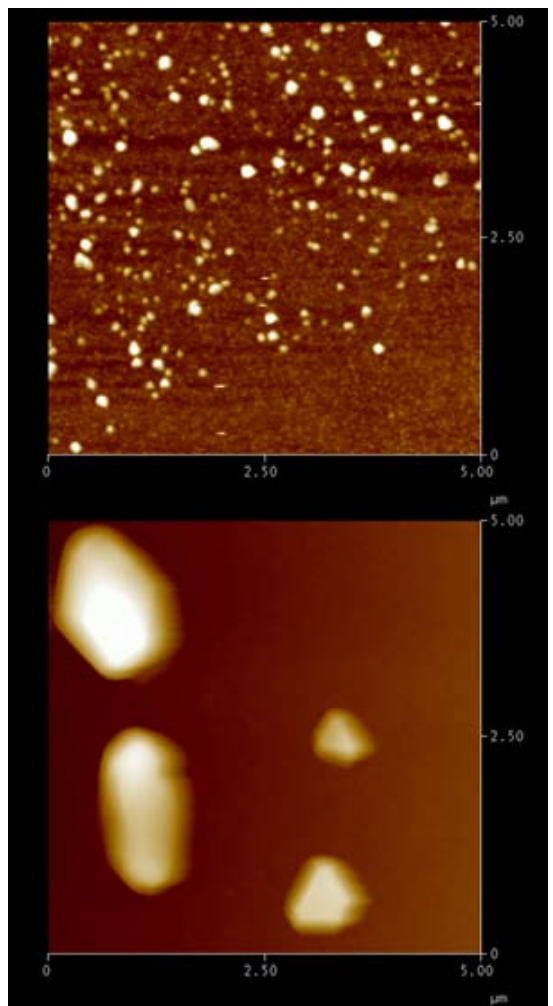

Fig. (5). $5 \mu \mathrm{m} \times 5 \mu \mathrm{m}$ images of Au crystal surface. Top: monomeric fibrinogen molecules prior to addition of thrombin. Bottom: fibrin polymer clot subsequent to thrombin addition.

\section{DISCUSSION}

In this paper, we prepared three different surfaces on top of the quartz crystals, representative of varying hydrophobicities, namely, hydrophilic polished gold, moderately hydrophobic PMMA, and a hydrophobic dodecanethiol SAM on polished gold. In theory, two separate types of clotting information can be assessed through the QCM measurements, namely, the amount of clotting proteins that adsorb onto a surface through $\Delta f$ of the crystal, and the extent of subsequent thrombin-induced cross-linking that will occur on the surface through analysis of $\Delta R$ of the crystal [11]. In order to simplify the analysis, only the final three proteins of the common coagulation pathway were used, namely fibrinogen, thrombin, and coagulation factor XIII. As fibrinogen is allowed to flow over the surface of the coated quartz crystal at some predetermined concentration and flow rate, an initial downward shift in the resonant frequency of the crystal can be observed. This frequency shift $(\Delta f)$ is proportional to the mass of materials (in this case fibrinogen) adsorbed to the crystal surface; furthermore, there is an initial 
increase in the resistance of the crystal, due to the viscoelastic properties of the adsorbed mass (materials with greater viscoelasticity will cause the crystal to exhibit greater resistance). Secondly, a solution containing thrombin and factor XIII is allowed to flow over the adsorbed layer of fibrinogen. At this point thrombin cleaves the fibrinogen to a fibrin monomer, followed by factor XIII induced cross-linking to form a clot. During this clot formation, no or very little additional proteins are adsorbed onto the crystal surface as coverage was already achieved by the fibrinogen in the first step; thus, there should be little to no additional change in the measured resonant frequency. Upon cross-linking, however, the fibrin monomer becomes more ideally rigid, thus a decrease in the resistance of the crystal can be observed. All of these measurements are recorded over time, such that the extent of fibrinogen absorption onto a surface and relative extent of subsequent cross-linking can be assessed. It should be noted that all three resistance vs. time graphs show a small sharp peak just before thrombin induced cross-linking. This is due to switching of delivery syringes, which causes a temporary decrease in the pressure at the crystal surface, thus causing a brief increase in the crystals resistance. Furthermore, the non-normal resistance increase which is most pronounced in figure three (bottom) occurs when a small gaseous bubble becomes temporarily lodged on the crystal surface; however the deviations are negligible in comparison to the resistance shifts caused by rigid mass loading.

The initial frequency decrease is representative of fibrinogen adsorption onto the crystal. The mass of the adsorbed layer can be calculated using the Sauerbrey equation,

$$
\Delta m=-C \cdot \Delta f
$$

where $C=17.7 \mathrm{ng} \mathrm{Hz}^{-1}$ for the $5 \mathrm{MHz}$ crystal we used [12]. The thickness of the adsorbed protein layer can then be calculated using

$$
\delta=\frac{3 \sqrt{3}}{\pi} \frac{C_{m}}{\rho_{f}}
$$

where $\delta=$ the layer thickness, $3 \sqrt{3} / \pi$ is the packing factor, $C_{m}$ is the plateau value of the adsorbed amount, and $\rho_{f}$ is the density of fibrinogen [12]. $C_{m}$ can be calculated from $\Delta \mathrm{m}$ by dividing it with the piezoelectrically active crystal area, 0.4 $\mathrm{cm}^{2}$ [13]. Inserting the mass value from eqn. 1 into eqn. 2 yields layer thicknesses for $\mathrm{Au}, \mathrm{PMMA}$. and $\mathrm{C}_{11} \mathrm{SH}$ of 35 $\mathrm{nm}, 62 \mathrm{~nm}$, and $51 \mathrm{~nm}$ respectively. Of course, the Sauerbrey equation overestimates the non-rigid mass loading by 2-6 times $[14,15]$. If the above thicknesses were overestimated by 4 times, the actual adsorbed layer thicknesses become 9 $\mathrm{nm}(\mathrm{Au}), 16 \mathrm{~nm}$ (PMMA) and $13 \mathrm{~nm}\left(\mathrm{C}_{11} \mathrm{SH}\right)$, respectively. As the hydrodynamic dimensions of the fibrinogen protein are $40-50 \mathrm{~nm}$ in length and $4-10 \mathrm{~nm}$ in width [16], these layer thicknesses indicate that protein packing on the gold surface is mostly side-on monolayer packing, and those on the PMMA and dodecanthiol surfaces are either in between side-on and end-on monolayer packings or in side-on multilayered packing.

This information also sheds light on the rates of protein adsorption on the various crystals over time. The frequency decrease on the gold surface is sharp to its plateau, at which point no further decrease is observed. This represents the protein saturating the surface with mono-layer coverage at which point no further adsorption can occur. In the case of PMMA and dodecanethiol, however, the frequency change is observed to increase indefinitely without reaching a time dependant plateau. This is most likely due to the continuous structural change from side-on to end-on conformation or multiple protein layer formation.

The AFM analysis was performed in order to demonstrate that the fibrinogen is indeed adsorbing to the crystal surfaces and then crosslinking upon thrombin addition. Saturation of the crystal surface with fibrinogen made it difficult to see any conformational changes in the protein (clot) formation; however, imaging a non-saturated crystal surface proved informative, as there is greater contrast between the proteins and crystal surface (Fig. 5). The AFM image taken prior to thrombin addition demonstrates that the proteins are adsorbing to the crystal surface in their monomeric form, while the large aggregates imaged subsequent to thrombin addition are representative of the clot formation. Addition of calcium ions to the thrombin solution was necessary, as calcium acts as a cofactor for coagulation factor XIII, which stabilizes the thrombin cleaved fibrin for polymerization.

Previous studies conducted using AFM indicate that fibrinogen will adsorb more readily and to a greater extent to hydrophobic surfaces than to hydrophilic surfaces [17]. The hydrophobicity of the surface, however, does appear to play an important role in the kinetics of protein adsorption, as saturation of adsorption on the hydrophilic gold surface (water contact angle $\theta=35^{\circ}$ ) occurs as early as $1,500 \mathrm{~s}$, while saturation on the moderately hydrophobic PMMA surface $(\theta$ $=62^{\circ}$ ) occurs at about 3,500 s, and not until $>5,000 \mathrm{~s}$ on the hydrophobic dodecanethiol SAM $\left(\theta=103^{\circ}\right)$. The resistance of the crystal is representative of the viscoelasticity of the loaded proteins, e.g. a softer loaded mass will exhibit greater increase in resistance. The resistance increase for the three model surfaces, gold, PMMA, and dodecanethiol SAM are $2.5 \Omega, 4 \Omega$, and $6 \Omega$ respectively, indicating that with increasing hydrophobicity of a given surface, the adsorbed fibrinogen film becomes less rigid. The most likely cause for this is slight denaturation of the adsorbed proteins due to affinity of the hydrophobic protein core to the hydrophobic material surface [18]. The decrease in resistance upon addition of the thrombin/factor XIII/Ca ${ }^{2+}$ solution is representative of clot formation as the adsorbed fibrinogen is crosslinked into the more rigid fibrin polymer. The resistance of the fibrinogen films on PMMA and dodecanethiol surfaces decreased at a greater extent upon cross-linking; four-fold over the gold surface. Actually the resistance became even lower than the initial value, indicating the layers of PMMA and dodecanethiol were somehow rigidified. This unusual phenomenon can be attributed an initial relative softness of the modified surfaces in comparison to gold, which fibrinogen cross-linking causes to become more rigid than the initial surface.

\section{REFERENCES}

[1] Dee KC, Puleo DA, Bizios R. Blood-biomaterial interactions and coagulation. An Introduction to Tissue-Biomaterial Interactions. John Wiley and Sons: Hoboken NJ 2002.

[2] Hansson KM, Johansen K, Wettero J, et al. Surface plasmon resonance detection of blood coagulation and platelet adhesion under 
venous and arterial shear conditions. Biosens Bioelectron 2007; 23(2): 261-8.

[3] Baker BR, Laiwalla AN, Yoon JY, Canavate J, Garrell RL. Adhesion and cohesion of mussel adhesive protein on glass and gold through protein removal studies. Polym Mater Sci Eng 2001; 85: 115-6.

[4] Saurbrey G. Verwendung von schwingquarzen zur wagung dunner schichten und zur mikrowagung. Z Phys 1959; 155(2): 206-22.

[5] Lucklum R, Hauptmann P. The quartz crystal microbalance: mass sensitivity, viscoelasticity and acoustic amplification. Sens Actuators B 2000; 70(1-3): 30-6.

[6] Evans-Nguyen KM, Fuierer RR, Fitchett BD, Tolles LR, Conboy JC, Schoenfisch MH. Changes in adsorbed fibrinogen upon conversion to fibrin. Langmuir 2006; 22(11): 5115-21.

[7] Choi KH, Friedt JM, Laureyn W, Frederix F, Campitelli A, Borghs G. Investigation of protein adsorption with simultaneous measurements of atomic force microscope and quartz crystal microbalance. J Vac Sci Technol B 2003; 21(4): 1433-6.

[8] Choi KH, Friedt JM, Frederix F, Campitelli A, Borghs G. Simultaneous atomic force microscope and quartz crystal microbalance measurements: Investigation of human plasma fibrinogen adsorption. Appl Phys Lett 2002; 81(7): 1335-7.

[9] Weber N, Pesnell A, Bolikal D, Zeltinger J, Kohn J. Viscoelastic properties of fibrinogen adsorbed to the surface of biomaterials used in blood-contacting medical devices. Langmuir 2007; 23(6): 3298-304.
[10] Tebbe D, Thull R, Gbureck U. Influence of spacer length on heparin coupling efficiency and fibrinogen adsorption of modified titanium surfaces. Biomed Eng Online 2007; 6: 31.

[11] Hedin J, Löfroth JE, Nydén M. Adsorption behavior and crosslinking of EHEC and HM-EHEC at hydrophilic and hydrophobic modified surfaces monitored by SPR and QCM-D. Langmuir 2007; 23(11): $6148-55$.

[12] Yoon JY, Park HY, Kim JH, Kim WS. Adsorption of BSA on highly carboxylated microspheres - quantitative effects of surface functional groups and interaction forces. J Colloid Interface Sci 1996; 177(2): 613-20.

[13] QCM200 and QCM25 Operation and Service Manual. Stanford Research Systems: Sunnyvale, CA 2004.

[14] Bizet K, Gabrielli C, Perrot H, Therasse J. Validation of antibodybased recognition by piezoelectric transducers through electroacoustic admittance analysis. Biosens Bioelectron 1998; 13(3-4): 259-69.

[15] Ha TH, Kim S, Lim G, Kim K. Influence of liquid medium and surface morphology on the response of QCM during immobilization and hybridization of short oligonucleotides. Biosens Bioelectron 2004; 20(2): 378-89.

[16] Doolittle RF. Fibrinogen and Fibrin. Sci Am 1981; 245(6): 126-35.

[17] Lin Y, Wang J, Wan LJ, Fang XH. Study of fibrinogen adsorption on self-assembled monolayers on $\mathrm{Au}(111)$ by atomic force microscopy. Ultramicroscopy 2005; 105(1-4): 129-36.

[18] Kim JH, Yoon JY. Protein adsorption on polymer particles. In: Hubbard A Ed, Encyclopedia of surface and colloid science. Marcel Dekker: New York, NY 2002; 4373-4381.

(c) Heinze and Yoon; Licensee Bentham Open.

This is an open access article licensed under the terms of the Creative Commons Attribution Non-Commercial License (http://creativecommons.org/licenses/ by-nc/3.0/) which permits unrestricted, non-commercial use, distribution and reproduction in any medium, provided the work is properly cited. 